\title{
Polarized Continuum Radiation from Stellar Atmospheres
}

\author{
J. Patrick Harrington \\ Dept. of Astronomy, University of Maryland \\ College Park, MD 20742, USA \\ email: jph@astro.umd.edu
}

\begin{abstract}
Continuum scattering by free electrons can be significant in early type stars, while in late type stars Rayleigh scattering by hydrogen atoms or molecules may be important. Computer programs used to construct models of stellar atmospheres generally treat the scattering of the continuum radiation as isotropic and unpolarized, but this scattering has a dipole angular dependence and will produce polarization. We review an accurate method for evaluating the polarization and limb darkening of the radiation from model stellar atmospheres. We use this method to obtain results for: (i) Late type stars, based on the MARCS code models (Gustafsson et al. 2008), and (ii) Early type stars, based on the NLTE code TLUSTY (Lanz and Hubeny 2003). These results are tabulated at http://www.astro.umd.edu/ $\sim j p h /$ Stellar_Polarization.html While the net polarization vanishes for an unresolved spherical star, this symmetry is broken by rapid rotation or by the masking of part of the star by a binary companion or during the transit of an exoplanet. We give some numerical results for these last cases.
\end{abstract}

Keywords. polarization, radiative transfer, astronomical data bases: miscellaneous, stars: atmospheres, binaries: eclipsing, planetary systems

\section{The Integral Equations}

By symmetry, radiation emergent from a plane-parallel atmosphere must be polarized either along the direction of the normal to the surface or perpendicular to it. Thus we need to consider only two Stokes parameters, $I$ and $Q$. These can be written in terms of a pair of source functions, $s_{\nu}\left(\tau_{\nu}\right)$ and $p_{\nu}\left(\tau_{\nu}\right)$ :

$$
\begin{gathered}
I_{\nu}(0, \mu)=\int_{0}^{\infty} s_{\nu}\left(\tau_{\nu}\right) e^{-\tau_{\nu} / \mu} \frac{d \tau_{\nu}}{\mu}+\left(\frac{1}{3}-\mu^{2}\right) \int_{0}^{\infty} p_{\nu}\left(\tau_{\nu}\right) e^{-\tau_{\nu} / \mu} \frac{d \tau_{\nu}}{\mu}, \\
Q_{\nu}(0, \mu)=\left(1-\mu^{2}\right) \int_{0}^{\infty} p_{\nu}\left(\tau_{\nu}\right) e^{-\tau_{\nu} / \mu} \frac{d \tau_{\nu}}{\mu}
\end{gathered}
$$

where $\mu=\cos \theta$, with $\theta$ being the co-latitude and $\tau_{\nu}$ is the monochromatic continuum optical depth.

Using the formal solutions of the transfer equations, we can write integral equations for $s_{\nu}\left(\tau_{\nu}\right)$ and $p_{\nu}\left(\tau_{\nu}\right)$ (Harrington, 1969, 1970):

$$
\begin{gathered}
s_{\nu}\left(\tau_{\nu}\right)=\left(1-\lambda_{\nu}\right)\left[\Lambda_{\tau_{\nu}}\left(s_{\nu}\right)+\frac{1}{3} M_{\tau_{\nu}}\left(p_{\nu}\right)\right]+\lambda_{\nu} B_{\nu}\left(\tau_{\nu}\right), \\
p_{\nu}\left(\tau_{\nu}\right)=\frac{3}{8}\left(1-\lambda_{\nu}\right)\left[M_{\tau_{\nu}}\left(s_{\nu}\right)+N_{\tau_{\nu}}\left(p_{\nu}\right)\right] .
\end{gathered}
$$

Here, $\Lambda_{\tau}$ is the familiar $\Lambda$-operator, $\left(1-\lambda_{\nu}\right)$ is the ratio of scattering to extinction, and 
$B_{\nu}\left(\tau_{\nu}\right)$ is the Planck function. We also need the additional operators $M_{\tau}$ and $N_{\tau}$ defined by

$$
M_{\tau}\{f(t)\}=\int_{0}^{\infty} f(t)\left[\frac{1}{2} E_{1}(|t-\tau|)-\frac{3}{2} E_{3}(|t-\tau|)\right] d t
$$

and

$$
N_{\tau}\{f(t)\}=\int_{0}^{\infty} f(t)\left[\frac{5}{3} E_{1}(|t-\tau|)-4 E_{3}(|t-\tau|)+3 E_{5}(|t-\tau|)\right] d t .
$$

In the above equations $E_{1}, E_{3}, E_{5}$ are the well-known exponential integrals.

\section{Matrix representation of the integral operators}

We choose an optical depth grid and assume that the source functions can be represented by cubic splines. We can then integrate the kernels of the transforms against cubic polynomials to obtain matrices which operate on the source functions to yield the $\Lambda_{\tau}$, $M_{\tau}$ and $N_{\tau}$ transforms. The transforms become matrix multiplications:

$$
\Lambda_{\tau_{i}}\left(s_{\nu}\right) \longrightarrow \sum_{j=1}^{N} \Lambda_{i j} s_{j} \quad \text { etc. }
$$

Here $N$ is the total number of optical depth points.

We assume that we have, from the stellar atmosphere models, values of the absorption $\kappa_{\nu}$ and the scattering $\sigma_{\nu}$ as a function of the monochromatic optical depth $\tau_{\nu}$ at the frequency of interest. We also need the temperature $T\left(\tau_{\nu}\right)$ so that we can evaluate $B_{\nu}\left(\tau_{\nu}\right)$. The ratio $\lambda$ is given by $\lambda_{\nu}=\kappa_{\nu} /\left(\kappa_{\nu}+\sigma_{\nu}\right)$. We choose a grid of optical depth points $\left(\tau_{\nu}\right)_{i}$ for $i=1, \cdots, N$ and evaluate the transforms $\Lambda_{\tau}, M_{\tau}$ and $N_{\tau}$ on this grid. The result is a system of linear equations for the unknown functions $s_{i}$ and $p_{i}$ :

$$
\left[\begin{array}{cc}
I_{i j}-\left(1-\lambda_{i}\right) \Lambda_{i j} & -\frac{1}{3}\left(1-\lambda_{i}\right) M_{i j} \\
-\frac{3}{8}\left(1-\lambda_{i}\right) M_{i j} & I_{i j}-\frac{3}{8}\left(1-\lambda_{i}\right) N_{i j}
\end{array}\right]\left[\begin{array}{c}
s_{i} \\
p_{i}
\end{array}\right]=\left[\begin{array}{c}
\lambda_{i} B_{i} \\
0
\end{array}\right]
$$

where $I_{i j}$ is an $N \times N$ identity matrix. Once a $\tau$-grid has been selected, the $\Lambda, M$ and $N$ matrices on the left hand side are fixed and need not be recomputed. We solve for $s_{i}$ and $p_{i}$ by standard methods of linear algebra. Then, using a similar matrix representation of the operator $E_{\mu}\{f(t)\}=\int_{0}^{\infty} f(t) e^{-t / \mu}(d t / \mu)$, we evaluate $I(\mu)$ and $Q(\mu)$ from the equations above.

For the results tabulated on our website, we have used $N=80$ depth points in the range $0<\tau_{i}<22$ with mostly logarithmic spacing. This leads to a system of 160 equations. We then evaluate $I(\mu)$ and $Q(\mu)$ for 159 values of $\theta$, the angle of the emergent radiation $(\mu=\cos \theta)$. The chosen angles are concentrated near $\mu=0$, where the polarization is greatest and is changing rapidly.

\section{Results}

For stars with effective temperatures between $2500 \mathrm{~K}$ and $8000 \mathrm{~K}$ and $\log (\mathrm{g})$ between 3 and 5, we have evaluated the polarization and limb darkening for 125 MARCS $\dagger$ model atmospheres with solar abundances, and also for grids with 1/10 and 1/100 solar abundances. Fig. 1 shows the polarization for 4000K models. The polarization decreases with increasing surface gravity. The upper curve is for $\log g=3.0$ and the lower is for $\log g=4.5$.

$\dagger$ MARCS website: http://marcs.astro.uu.se/ 


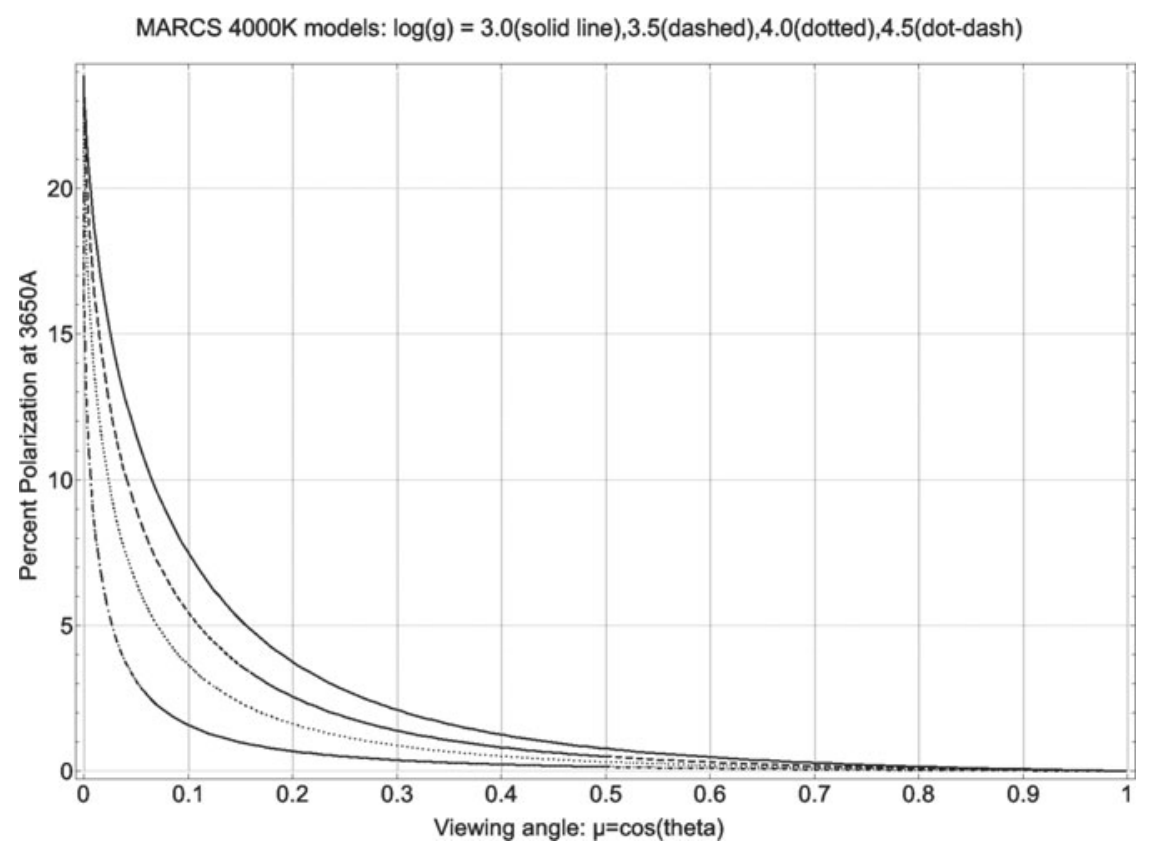

Figure 1. Continuum polarization from cool stellar atmosphere models.

For hot stars we made use of the NLTE results of the TLUSTY $\ddagger$ code. We tabulate results for 43 models with temperatures between 15,000K and 50,000K, solar abundances, and a range of gravities. We also give a few 1/10 solar models. The hot stars generally exhibit less polarization. One curious feature is that the plane of polarization may shift by 90 degrees (see Fig. 2).

Between $8000 \mathrm{~K}$ and $15,000 \mathrm{~K}$, neither the MARCS or TLUSTY models are available. However, there are the LTE STERNE $\dagger$ models of C.S. Jeffery which span the 800K $50,000 \mathrm{~K}$ range. But these models only provide the opacity at one wavelength, so that it is only straightforward to evaluate the polarization and limb darkening at 4000A. Fig. 2 shows that the STERNE and TLUSTY results for a $35,000 \mathrm{~K}, \log \mathrm{g}=3.5$ model are very similar.

Recently, Kostogryz \& Berdyugina (2015) showed results for 4500K stars which we can compare with our MARCS results. As shown in the Fig. 3, their values are quite similar, but about $15 \%$ lower than ours.

The main deficiency of these calculations is the neglect of spectral lines, since there may be little or no continuum over large parts of the spectrum. This tends to be the case at the short wavelength end of the spectrum, which, unfortunately, is where the maximum of the continuum polarization occurs. In the case of the sun, studies of the "2nd solar spectrum" show that the majority of the (weak) absorption lines are depolarizing relative to the continuum. This suggests that we may model the effects of line blanketing by modifying a spectrum synthesis code to output the depth-dependent values of $\kappa_{\nu}\left(\tau_{\nu}\right)$ and $\sigma_{\nu}\left(\tau_{\nu}\right)$, where $\kappa_{\nu}$ includes the line absorption. This must be done for a sufficiently fine frequency grid to resolve the individual spectral lines. We have tried this with the SYNSPEC code applied to some TLUSTY models, outputting data for $>10^{5}$ frequency points. We use the matrix operator method presented above to compute $I(\mu)$ and $Q(\mu)$

$\ddagger$ TLUSTY website: http://nova.astro.umd.edu/

$\dagger$ STERNE website: http://star.arm.ac.uk/ csj/models/Grid.html 


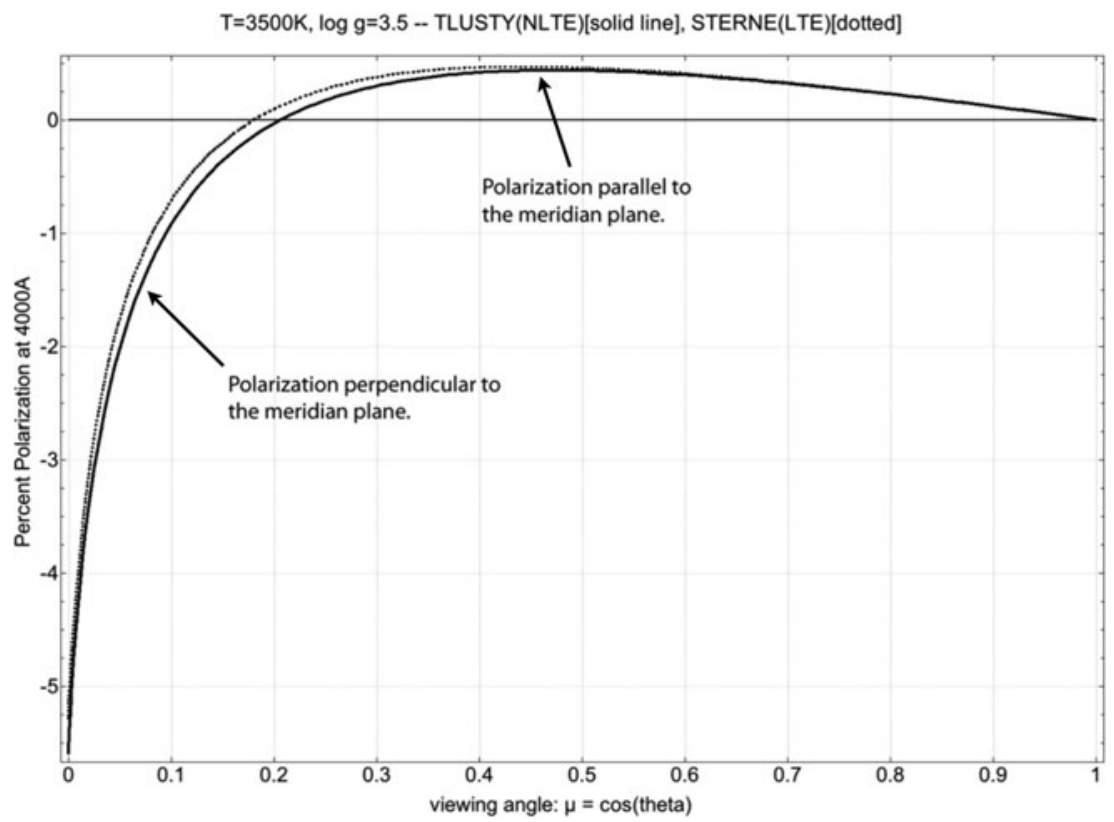

Figure 2. Continuum polarization from hot stellar atmosphere models.

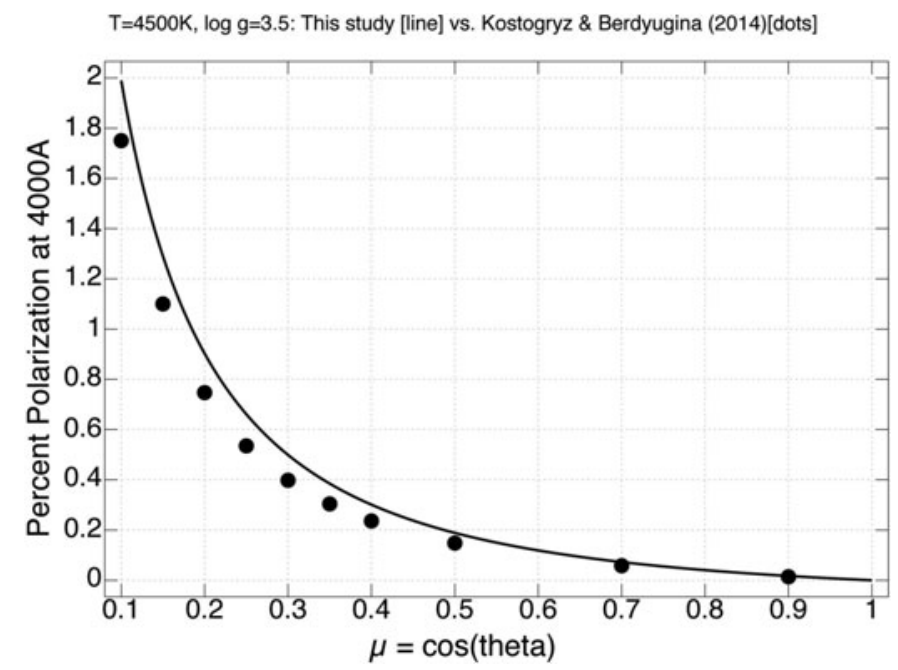

Figure 3. Comparison with values from Kostogryz and Berdyugina (2015).

at each frequency point. We may then average the Stokes parameters over a wavelength band to obtain the expected net polarization. We give some results on our webpage (http : //www.astro.umd.edu/ jph/Stellar_Polarization.html). For example, for one star, in the line-rich region around $1600 \mathrm{~A}$, we find a reduction in the average polarization of $\sim 30 \%$ compared to the continuum-only computation.

\section{Applications: Observability of the Polarization}

Since the polarization from an unresolved spherical star cancels out, we must look for situations where the symmetry is broken. This would include: 
Transit of Kepler-16A by star B [dashed line] and by planet $b$ [solid line]

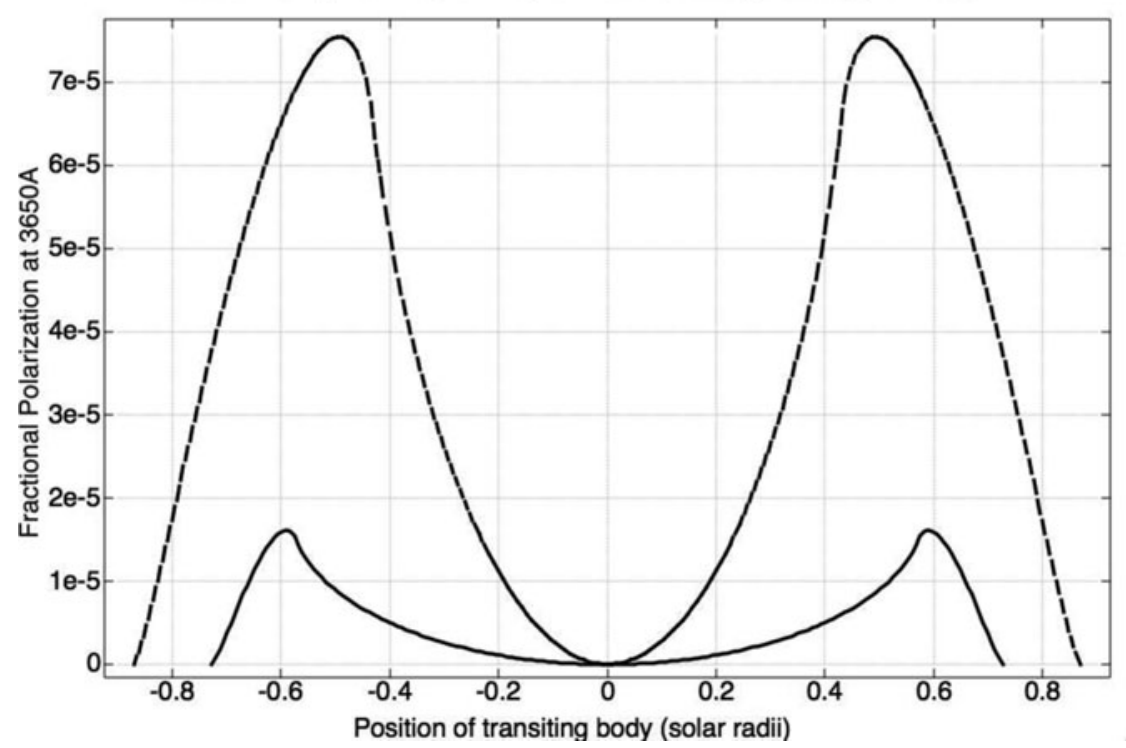

Figure 4. Polarization from the transit of a companion star and a planet.

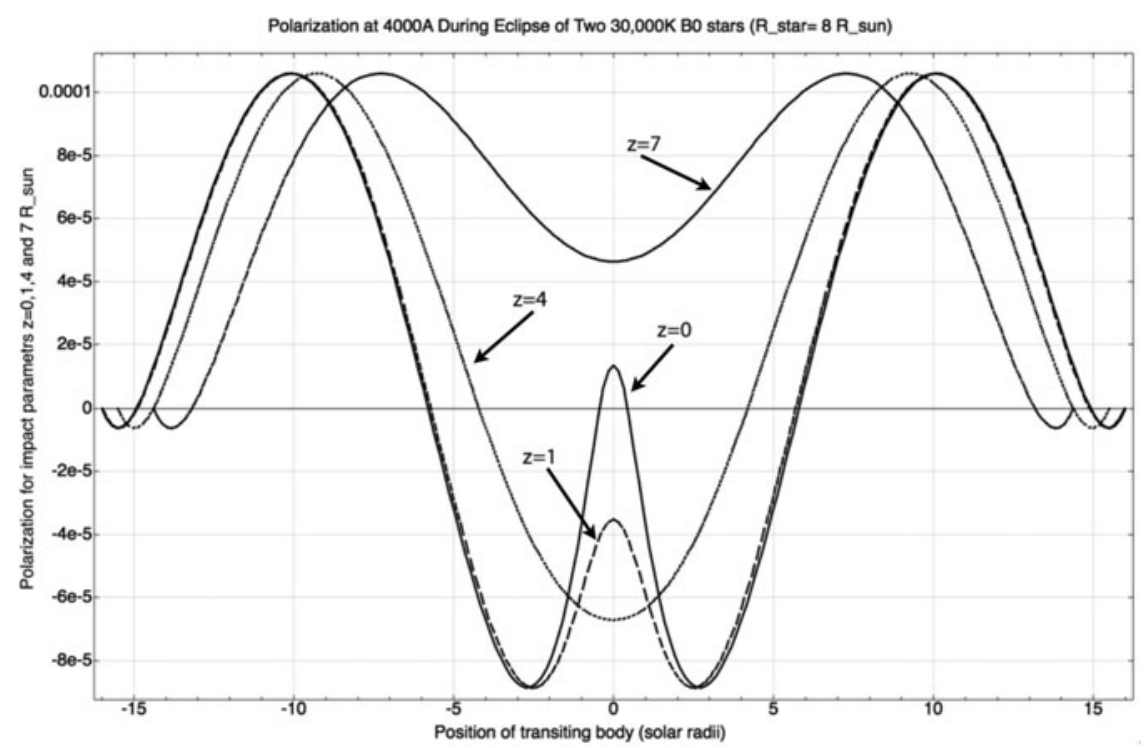

Figure 5. Polarization from the eclipse of two identical B0 stars. The curves represent different orbital inclinations, parameterized by the impact parameter $\mathrm{z}$ (the closest approach of the projected star centers). The solid curve $(\mathrm{z}=0)$ is the polarization seen by an observer in the orbital plane.

- The effects of rapid rotation or tidal distortion.

- Large starspots or giant convective cells.

- The masking of part of the stellar disk by a transiting stellar or planetary companion.

For this last case, we have written code to evaluate the net polarization for stars with part of their disk blocked by a star or planet, using the tables of $I(\mu)$ and $Q(\mu)$ discussed above. 
In Fig. 4 we show results for the star Kepler-16A, $\mathrm{T}=4500 \mathrm{~K}, \log \mathrm{g}=4.5$, and a radius of $0.65 R_{\odot}$. It is a binary with a small companion with a radius of $0.22 R_{\odot}$ and low luminosity, and in addition has a transiting planet with a radius of $0.0775 R_{\odot}$. The transit of the exoplanet produces a polarization signal of a bit over 1e-5, while the transit of the companion star might give a fractional polarization of $7 \mathrm{e}-5$.

Fig. 5 illustrates a different situation, the mutual eclipse of two identical $\mathrm{T}=35,000 \mathrm{~K}$, $\log (\mathrm{g})=3.5$ stars. Because, as noted above, the polarization of light from the center of the disk is polarized at right angles to the light from the limb, the variation of polarization during the eclipse is more complicated.

\section{References}

Gustafsson, B., Edvardsson, B., Eriksson, K., Jørgensen, U. G., Nordlund, Å., \& Plez, B. 2008, $A \& A 486,951$

Harrington, J. P. 1969, Ap.Lett. 3, 165

Harrington, J. P. 1970, APESS 8, 227

Kostogryz, N. M. \& Berdyugina, S. V. 2015, A\&A 4575 , A89

Lanz, T. \& Hubeny, I. 2003, ApJS 146, 417 